\title{
Variations
}

Variations

Revue internationale de théorie critique

$21 \mid 2018$

L'industrie de la culture : version originale

\section{La Théorie critique au XXIème siècle}

Oskar Negt et Alexander Kluge

Traducteur : Alexander Neumann

\section{(2) OpenEdition}

Journals

Édition électronique

URL : http://journals.openedition.org/variations/961

DOI : 10.4000/variations. 961

ISSN : 1968-3960

Éditeur

Les amis de Variations

Référence électronique

Oskar Negt et Alexander Kluge, « La Théorie critique au XXlème siècle », Variations [En ligne], 21 | 2018, mis en ligne le 05 avril 2018, consulté le 19 avril 2019. URL : http://journals.openedition.org/ variations/961 ; DOI : 10.4000/variations.961

Ce document a été généré automatiquement le 19 avril 2019.

Les ami•e•s de Variations 


\title{
La Théorie critique au XXIème siècle
}

\author{
Oskar Negt et Alexander Kluge
}

Traduction : Alexander Neumann

\section{NOTE DE L'ÉDITEUR}

Nous remercions Oskar Negt de son aimable autorisation pour l'édition française de cet extrait, paru dans Massverhältnisse des Politischen, in Der unterschätzte Mensch, Band 1, Zweitausendeins, 2001, pp.991-97.

Qu'est-ce qui fonde nos jugements, pour nous permettre de distinguer le vrai du faux? Comment nous décidons ce que nous recherchons dans la vérité, ou quelle vérité nous intéresse? Quelle part de ce qui nous intéresse nait de nos besoins impérieux? Comment savoir ce qui nous est nécessaire? Dit autrement : comment savons-nous si notre curiosité à connaitre le monde va s'avérer suffisant à la fin? Quelle sorte de Tribunal allons nous reconnaître pour trancher ces questions qui touchent à la critique, c'est-à-dire notre faculté de juger, de produire la distinction? Nous avons besoin d'une masse de facultés de juger, qui augmente avec les masses d'antécédents historiques, de réalités qui nous précèdent, réalités auxquelles nous sommes tous confrontés.

\section{La Théorie critique}

2 L'Ecole de Francfort - dont nous sommes - s'est d'abord focalisée sur des processus politiques intervenus depuis la première guerre mondiale. Cette approche de recherche a permis d'élaborer des positions claires, face à la montée du fascisme, tout en gardant une distance envers ces penchants du socialisme qui favorisent la construction d'appareils, ce qui a permis de contrer le cannibalisme politique que ce processus a déclenché. Pendant l'émigration et l'exil, la Théorie critique jette des ponts entre les deux rives de l'océan atlantique, puis, de retour en Allemagne fédérale, elle apporte tout un arsenal d'expériences fondamentales et nouvelles. 
3 Les philosophes classiques exposèrent les prolégomènes de leur pensée à travers des cours, la rédaction de traités, qu'ils développèrent alors par la méditation, en toute liberté et toute solitude, un travail par lequel ils ouvraient littéralement des chantiers. Emmanuel Kant parle de l'architecture de la raison, il voit les penseurs comme des travailleurs de chantier, qui interviennent sur un bâtiment en construction, comparable à la tour de Babel, pendant qu'ils érigent des maisons et des appartements susceptibles d'accueillir l'expérience des être humains d'un point due vue pratique. ${ }^{1}$ Jusqu'à l'époque de Nietzsche, d'Heidegger ou encore de Theweleit un genre de philosophe qui agit en entrepreneur individuel domine la scène, un peu comme Kant et Hamann agissaient à Kaliningrad à leur époque. Leurs médias respectifs sont la discussion générale au sein de l'espace public bourgeois, ou la politique universitaire.

En revanche, Horkheimer et Adorno travaillent, eux, au sein d'un institut ou en réseau (ce qui n'enlève pas leur individuation au sens classique du terme). La dialectique de la raison (Dialektik der Aufklärung) émane d'une écriture commune. Les études sur l'autorité et la famille (Autorität und Familie) sont le fruit d'un travail collectif, mené par un grand nombre de personnes. La même chose vaut pour leur revue, la Zeitschrift für Sozialforschung, qui offre une plateforme qui met en relation des esprits aussi différents que Walter Benjamin, Franz Neumann, Karl Korsch. Il est révélateur que Walter Benjamin travaille à la Bibliothèque nationale à Paris, ce qui lui offre bien plus d'interconnexions qu'un bureau personnel. $^{2}$

5 La Théorie critique combine une forte mise en réseau, l'interdépendance de ses projets critiques singuliers, ainsi que l'organisation de son indépendance intellectuelle, qui doit être maximale. Cet ensemble n'obéit à aucun parti, ni aucune formation sociale, et échappe à une définition circonscrite au cadre national. Elle noue des fils entre la psychanalyse, la recherche sociale empirique, l'historiographie, l'analyse politique et même dans sa ramification habermassienne - la reconstruction de grands ensembles théoriques.

\section{Modalités, échelles et diffusion de la PENSEE (auto- réflexive) au début du 21 ème siècle}

6 La question de savoir ce qu'est la pensée ne trouvera ni de réponse dans les statistiques ni de définition dans le langage courant. Les changements et métamorphoses qui se font jour dans les échelles de la pensée, ou dans la théorie de l'activité cognitive, ne ressortent nettement que si nous observons avec un certain recul le grand cycle des 300 dernières années. Les procès de travail de l'intelligence ne sont pas principalement portés par les courants d'idée qui se distinguent par des déclarations d'intention, des manifestations professionnelles pointues ou encore par des performances individuelles marquantes, mais ils déploient plutôt leur pertinence maximale aux marges du mainstream. Ces affluents charrient du flou, des éléments d'auto-régulation, l'absence d'intention précise, et même de l'inconscience et de l'aveuglement. Pour bien cerner l'enjeu, il convient de poser la question du point de vue du Sirius de Voltaire, un observateur complètement étranger à notre culture mondaine, en appliquant à la situation actuelle sa méthode de la compréhension par l'abstraction. 


\section{La formation des Lumières à partir des émotions et des affaires du 17ème siècle}

7 Max Horkheimer a reconstruit la pratique de l'intelligentsia moderne, en partant de la période historique au 17ème siècle qui se caractérise alors par la puissante percée du mode de production bourgeois. C'est dans ce contexte qu'émerge une prise de position qui exprime la confiance en soi : cogito ergo sum. La nouvelle manière intelligente de concevoir la terre entière à partir de l'idéal des affaires économiques de chacun va caractériser un nouveau mode travail, qui se développe de manière particulièrement expansive et agressive. Cela se manifeste dans la comptabilité et les sciences de la gestion, tout autant que dans le colonialisme, dans la définition cartographique du monde, dans l'art des jardins publics et l'élevage des tulipes, ou encore dans les corps de fonctionnaires des Etats-nations, dans la philosophie, dans les droits de l'Homme, et aussi dans la quête intime du bonheur. La traite des esclaves et l'émancipation sont des processus qui ont lieu simultanément. Les traits marquants de cette nouvelle mentalité, qui se fonde sur un mode d'échange mondial et unique des intelligences, s'inscrivent dans la fixation individuelle de la conscience de soi, dans l'universalité, dans le rapport à l'utilité pratique de toute chose, et surtout dans le dualisme, qui affirme un sujet pensant qui s'érige en face des objets qu'il s'agit de transformer ou de s'approprier.

\section{Qu'est-ce que l'industrialisation de la conscience?}

Depuis plus de 300 ans, l'Europe est la scène d'une lutte violente entre la subjectivité et l'objectivité (avec des répercussions et formes qui varient en fonction des cinq continents), une lutte dont l'avancée des fronts bascule régulièrement. La littérature décrit le cours de ces évènements. Dans Les années d'apprentissage de Wilhelm Meister, le sujet s'empare du monde, par le biais d'un imposant voyage d'études, avant d'être pris d'effroi devant sa propre omnipotence. ${ }^{3}$ Dans Berlin, Alexanderplatz, écrit au lendemain de la première guerre mondiale, Alfred Döblin met en scène des marteau-pilons qui vrombissent dans la tête de Franz Biberkopf, dans un Berlin en pleine mutation. Le monde objectif s'empare du noyau subjectif. ${ }^{4}$ Les résidus du fantasme d'omnipotence se retournent alors pour provoquer l'impuissance, le ressentiment et finalement un assassinat.

En ce sens, les relations proportionnelles entre le subjectif et l'objectif se déplacent, à plusieurs reprises. L'élément décisif n'est pas seulement le changement qui a lieu, mais le lieu du changement qui esquisse une ligne de soudure, ce que Marx appelle le subjectifobjectif. Est-ce que la ligne de soudure reste perméable, riche? Est-ce qu'elle désigne la conscience (la critique)? Est-elle spontanée (idiosyncratique)? Est-elle auto-régulée?

Des catégories comme la liberté, l'auto-détermination, la cohésion sociale, le droit collectif, le savoir, la réflexion, dépendent toutes de pareilles lignes de soudure, qui doivent être examinées et entretenues en permanence. Jusqu'alors, l'industrialisation de la conscience ne s'est pas pleinement déployée à travers ce processus subjectif-objectif. Elle en est restée à l'état de projet. Certes, la subsumption des êtres humains sous le capital, et sous la discipline industrielle, s'est emparée de la pensée, des corps, des modes de vie. La subjectivité, en tant que noyau du travail cognitif, continue cependant à 
s'alimenter d'un genre de nature pré-industrielle. En revanche, le processus de l'industrialisation a largement découragé la pensée (en lien avec les frustrations liées aux guerres).

11 Si notre observateur venu de Sirius devait évaluer la somme d'intelligence que les sujets exercent aujourd'hui de manière autonome, en leur propre nom et selon leur propre responsabilité, en comparaison avec la masse d'intelligence qu'ils déploient pour d'autres, sous la forme de prestations de service ou d'efforts qui dépendent de la décision d'autres, il devrait constater que $90 \%$ de leurs activités concernent le second cas.

\section{L'intelligence en tant que prestation de service}

En pratique, le gros des efforts intellectuels se produisent dans le domaine professionnel, dans des think tanks, des fondations scientifiques, des agences, des médias, des lobbies, dans les départements de recherche \& développement, dans les mécanismes de contrôle managériaux, dans les rapports salariaux, dans des fonctions du spectacle, et dans d'autres formes dépendantes ou interdépendantes.

Ce qui est plus grave encore, devrait-on répondre à notre invité de Sirius, est l'état des activités cognitives non-professionnelles. Est-ce que les êtres humains, pourrait demander alors l'invité de Sirius s'il voulait persévérer, sont-ils intéressés par la vérité ou la pensée, au fond? Leur talent principal ne résiderait-il pas plutôt dans la capacité à s'enrôler dans des cocons, qui leur permettent de survivre et de se protéger contre le courant froid qui traverse le monde? Que ce soit par la création de religions, d'illusions, par l'oubli, le divertissement ou d'autres déplacements de terrain encore? Seraient-ils ces êtres vivants qui produisent des illusions, dont par Friedrich Nietzsche? Il est vrai que la recherche d'un équilibre personnel interne nécessite un effort intellectuel, vivace et massif, qui ne donne aucune impulsion à l'émancipation, mais qui maintient au contraire la dépendance. Cela s'applique aussi à la quête d'une économie individuelle équitable, l'auto-protection, l'apprentissage quotidien, la recherche d'un bonheur personnel. Partant de la matrice des subjectivités, dans leur constitution réelle au 21ème siècle, l'invité de Sirius devrait conclure que nos performances de jugement critique exposent avant tout notre cruel manque de ressources sociales à l'échelle planétaire.

La critique, c'est-à-dire la capacité de juger et de distinguer, doit faire face à cette praxis bien réelle de l'activité intelligente des humains, ce qui signifie qu'elle doit prendre pied à l'intérieur de cette pratique. Elle n'existe pas en tant qu'entité séparée, qui ferait face au processus social depuis l'extérieur. Pour cette raison, la critique reste impuissante si elle se contente de s'exprimer à travers des écrits, comme au 18ème siècle, ou si elle se fie seulement aux discussions qui sillonnent l'espace public, dont l'évolution est très lente. La critique doit ainsi forger ses propres instruments pratiques qui interviennent dans la praxis, sous la forme de contre-productions. ${ }^{5}$

Depuis 1865 existent des ascenseurs qui montent jusqu'au 40ème étage et davantage. Les gratte-ciels poussent dans les métropoles depuis cette date. A New York, ce genre d'architecture se perpétue de la manière la plus marquante : une espèce de BABYLONE multiculturelle qui est tolérante par nécessité, un symbole du "principe de la ville" (Richard Sennett). 

l'évaluation critique. Dans son livre Debt of honor de 1994, Tom Clancy avait décrit le crash d'un avion de ligne, détourné par des terroristes, qui s'échoue sur le Capitole. Le président des Etats Unis et la plupart des membres de son administration meurent. L'expert en sécurité Ryan, vice-président en attente de confirmation, rétablit l'ordre pour éviter la destruction des USA. Il s'agit un ROMAN qui ne pourrait plus être rédigée de cette même manière, après les attentats du 11 septembre 2001.

\section{NOTES}

1. Voir les passages sur le chantier et la construction dans Negt/Kluge, Geschichte und Eigensinn (1980), Steidl, 2016, p.247.

2. Si l'activité d'un philosophe correspond au mode de production d'un jardinier, d'un artisan ou d'un boutiquier, l'Institut de recherche sociale prend déjà la forme d'une manufacture.

3. Voir aussi la mort de Mignion dans Goethe, Les affinités électives, et son Faust II, 5ème acte.

4. Cela forme la base du roman de Musil, L'homme sans qualités.

5. Negt/Kluge, Geschichte und Eigensinn (Histoire et subjectivité rebelle), Zweitausendeins, (1980) 2016, pp.450.

\section{INDEX}

Mots-clés : Ecole de Francfort, Théorie critique, Walter Benjamin, Albert Einstein, Industrie de la conscience

\section{AUTEURS}

\section{OSKAR NEGT}

Professeur em. à l'Université d'Hanovre 


\section{ALEXANDER KLUGE}

Essayiste, réalisateur et producteur 